\title{
Differences on vascular reactivity and RNAm expression of Renin-Angiotensin and Kinin-Kallikrein systems components on acute and chronic myocardial infarction in male and female Wistar rats
}

\author{
Jazmin Flores-Monroy ${ }^{1}$, Diana Ramirez-Hernandez ${ }^{1,2}$, Diego Lezama-Martinez ${ }^{1,2}$, Luisa Martinez- \\ Aguilar $^{1}$
}

${ }^{I}$ Biochemistry and Pharmacology, Universidad Nacional Autonoma de Mexico, Mexico, ${ }^{2}$ Instituto Politecnico Nacional, Escuela Superior de Medicina, Mexico

Actually relative expression of mRNA of RAS and KKS had been widely investigated on males at acute or chronic myocardial infarction; also transcriptional changes had not been related with vascular activity. The aim of this study was to evaluate the relative mRNA expression of the ACE, ACE2, AT1, AT2, B1 and B2 and vascular reactivity to Ang II during the evolution of myocardial infarction. Methods: 80 male and 80 female Wistar rats were used and grouped as: 1) Sham; Coronary occlusion groups: 2) $48 \mathrm{~h}$; 3) 1 week; 4) 2 weeks; 5) 3 weeks; 6) 4 weeks. To all groups the relative mRNA expression of the ACE, ACE2, AT1, AT2, B1 and B2 receptors of the left ventricle by real time PCR were obtained. Also infarct area, cardiac hypertrophy, systolic and diastolic blood pressure, cardiac frequency and Ang II vascular reactivity in the absence and presence of valsartan $(200 \mathrm{nM})$ on aortic rings were evaluated. Results: Both sexes showed significant increase of ACE, ACE2, AT1, AT2, B1 and B2 mRNA expression in males at 3 weeks of CO and females at $48 \mathrm{~h}$ and 2 weeks and the last expressed in less amount all components. Infarct areas were calculated between 30-40\% in all groups, no changes were observed in hypertrophy index on female rats while in males there was an increase at 2, 3 and 4 weeks. A decrease in systolic and diastolic pressure in male rats at 3 weeks of $\mathrm{CO}$ was found. Vascular reactivity to Ang II was increased on male rats aortic rings during 4 weeks; on females, this effect is diminished since $48 \mathrm{~h}$ until reaching sham group values at 2 weeks of MI and the incubation with valsartan generated greater response reduction to Ang II in males than females. Conclusion: Our findings indicate that Renin-Angiotensin and Kinin-Kallikrein systems components are over expressed on male than female rats that could be related with functional and morphological changes on development of myocardial infarction. 